Cahiers $d u$ MONDE RUSSE

\section{Cahiers du monde russe}

Russie - Empire russe - Union soviétique et États indépendants

48/2-3 | 2007

Les résonances de 1905

\title{
Le Saint-Siège face à la révolution de 1905
}

Entre réflexes conservateurs, réformisme et enthousiasme missionnaire

Laura Pettinaroli

\section{(2) OpenEdition}

1 Journals

Édition électronique

URL : https://journals.openedition.org/monderusse/9018

DOI : 10.4000/monderusse. 9018

ISSN : $1777-5388$

Éditeur

Éditions de l'EHESS

\section{Édition imprimée}

Date de publication : 15 avril 2007

Pagination : 449-462

ISBN : 978-2-7132-2147-7

ISSN : $1252-6576$

Référence électronique

Laura Pettinaroli, « Le Saint-Siège face à la révolution de 1905 », Cahiers du monde russe [En ligne], 48/2-3 | 2007, mis en ligne le 01 janvier 2007, consulté le 04 septembre 2022. URL : http:// journals.openedition.org/monderusse/9018; DOI : https://doi.org/10.4000/monderusse.9018 
chercher : repérer : avancer

Cet article est disponible en ligne à l'adresse :

http://www.cairn.info/article.php?ID REVUE=CMR\&ID NUMPUBLIE=CMR 482\&ID ARTICLE=CMR 4820449

Le Saint-Siège face à la révolution de 1905. Entre réflexes conservateurs, réformisme et enthousiasme missionnaire

par Laura PETTINAROLI

\section{Editions de l'EHESS | Cahiers du monde russe}

2007/2-3 - Vol 48

ISSN 1252-6576 | ISBN 9782713221477 | pages 449 à 462

Pour citer cet article :

—Pettinaroli L., Le Saint-Siège face à la révolution de 1905. Entre réflexes conservateurs, réformisme et enthousiasme missionnaire, Cahiers du monde russe 2007/ 2-3, Vol 48, p. 449-462.

Distribution électronique Cairn pour les Editions de l'EHESS.

(C) Editions de l'EHESS. Tous droits réservés pour tous pays.

La reproduction ou représentation de cet article, notamment par photocopie, n'est autorisée que dans les limites des conditions générales d'utilisation du site ou, le cas échéant, des conditions générales de la licence souscrite par votre établissement. Toute autre reproduction ou représentation, en tout ou partie, sous quelque forme et de quelque manière que ce soit, est interdite sauf accord préalable et écrit de l'éditeur, en dehors des cas prévus par la législation en vigueur en France. Il est précisé que son stockage dans une base de données est également interdit. 


\title{
LE SAINT-SIÈGE FACE À LA RÉVOLUTION DE 1905
}

\author{
Entre réflexes conservateurs, réformisme \\ et enthousiasme missionnaire
}

Si, d'une façon générale, l'étude de la révolution russe de 1905 a été relativement négligée, cette observation vaut aussi pour l'historiographie des relations entre le Saint-Siège et la Russie. En effet, dans la plupart des études, c'est la révolution de 1917 et ses suites qui ont absorbé l'essentiel de l'attention ${ }^{1}$. Pourtant, les événements de 1905 modifient profondément les conditions d'existence du catholicisme (liberté religieuse, conversions de masse au catholicisme latin dans les provinces occidentales de l'Empire, prémices d'un catholicisme russe de rite oriental).

Bon poste d'observation des enjeux, encore méconnus, du catholicisme dans l'Empire russe, le Saint-Siège témoigne par ailleurs d'un intérêt missionnaire nouveau pour ce pays. En effet, depuis les années 1850-1860, s'est développé dans les milieux catholiques un effort de connaissance du monde russe, lié au mouvement orientaliste (revues d'érudition, Bibliothèque slave des jésuites russes résidant à Paris), soutenu par une tension proprement religieuse (prière pour la Russie dont le père Schouvaloff et la congrégation des barnabites sont les fers de lance). Ces catholiques de la fin du XIX ${ }^{e}$ siècle envisagent deux cas de figure «d'ouverture » de la

1. L'ouvrage qui demeure probablement la meilleure synthèse sur les relations Vatican-Russie au XXe siècle commence son étude en 1917 (Hansjakob Stehle, Die Ostpolitik des Vatikans, 1917-1975, Munich-Zurich : Piper, 1975). De même, l'une des meilleures études réalisées à partir des archives vaticanes sur la révolution russe de 1917 rejette dans le « lieu commun » la vision de la Russie par le Saint-Siège avant la révolution de 1917 (Roberto Morozzo della Rocca, Le nazioni non muoiono. Russia rivoluzionaria, Polonia indipendente e Santa Sede, Bologne : Il Mulino, 1992). Enfin, le lieu le plus dynamique de diffusion des recherches sur ce thème depuis la fin des années 1990 (les quatre colloques organisés conjointement par le Comité pontifical des sciences historiques et l'Institut d'histoire universelle de l'Académie des sciences de Moscou) n’a porté aucune attention spécifique à la révolution de 1905. 
Russie : ou bien un changement religieux sous la forme d'une réunion de l'Église russe à Rome (auquel cas le catholicisme deviendrait un rempart contre la révolution et une force de modernisation respectueuse des traditions ${ }^{2}$ ) ou bien un changement politique ouvrant la voie à des changements religieux (dans ce cas, le catholicisme profiterait d'une révolution venant rompre le système autocratique et le principe de religion d'État). Dès lors, on peut se demander si cette attente, parfois messianique, de changements en Russie ne va pas entrer en confrontation avec la culture réactionnaire élaborée à Rome depuis la Révolution française.

C'est pourquoi, il importe d'étudier précisément la réception de la révolution de 1905 par le Saint-Siège, en sollicitant les archives vaticanes (archives de la Secrétairerie d'État et de la Congrégation des affaires ecclésiastiques extraordinaires, essentielles pour les questions de politique internationale $)^{3}$, mais également des sources imprimées, en privilégiant le quotidien officieux de la papauté qu'est L'Osservatore Romano et le bimensuel des jésuites romains La Civiltà Cattolica. Cette presse est utile pour saisir, jour après jour, la sensibilité des milieux romains, proches des centres décisionnels du Saint-Siège.

La révolution russe de 1905 suscite au Vatican une succession d'espoirs et de déceptions, qu'il convient d'aborder chronologiquement : avril 1905, décembre 1905 et l'été 1907 constituant des points d'inflexion.

\section{Espérances et prudence de Rome face à une révolution en gestation (fin 1904 - avril 1905)}

En attente de changements, Rome n'hésite pas à encourager Nicolas II sur la voie des réformes et à le soutenir sur le front de l'opinion occidentale.

\section{L'attente de changements face à une situation bloquée}

En effet, malgré la reprise de relations diplomatiques en 1894 et la sympathie des papes pour Nicolas II (nette depuis l'invitation de 1899 à la conférence de La Haye), les points de blocage entre le Saint-Siège et la Russie sont nombreux à l'aube de 1905. Certains problèmes sont récurrents (longues vacances des sièges épiscopaux, limitations des communications avec Rome), d'autres sont plus récents comme le conflit sur les examens dans les séminaires de Pologne. C'est ainsi que, malgré un accord bilatéral entre Russie et Saint-Siège en 1897, le gouvernement russe avait modifié administrativement, en 1900 et en 1902, les modalités de

2. C'est du moins la thèse développée par Jean Gagarin dans La Russie sera-t-elle catholique ?, P.: Ch. Douniol, 1856: la quatrième et dernière partie de l'ouvrage étant intitulée « Catholicisme ou révolution ».

3. Abréviations: Archives Secrètes du Vatican (ASV), Secrétairerie d'État (Segr. Stato), Affaires ecclésiastiques extraordinaires (AES); rubrique (rub.), dossier (pos.), fascicule (fasc.), foglio (f.) 
l'examen de russe et ajouté un examen pour permettre au séminariste diplômé d'obtenir un office ecclésiastique. De ce fait, certains séminaristes diplômés ne pouvaient entrer en charge ${ }^{4}$.

\section{Un contexte de « pacification religieuse $»^{5}$ qui invite à la modération}

Malgré ces blocages, une évolution de la législation religieuse est bien en cours depuis 1903, à laquelle le Saint-Siège ne se montre pas indifférent. L'oukase du 26 février (11 mars) 1903 où « figure pour la première fois dans un texte officiel la tolérance religieuse $»^{6}$ suscite réflexion au Saint-Siège alors que s'opère la transition entre Léon XIII et Pie X (été 1903). C'est par une lettre personnelle particulièrement amicale du 16 décembre 1904 (en préparation depuis le printemps 1904), que Pie X félicite l'empereur de cet oukase dont les paroles « Nous ont fait concevoir les plus douces espérances pour une parfaite pacification religieuse $»^{7}$.

Cette lettre croise quasiment une nouvelle avancée du tsar sur la question de la tolérance religieuse. En effet, au sein de l'oukase annonciateur de réformes du 12/ 25 décembre $1904^{8}$, le paragraphe 6 manifeste la volonté de l'empereur de réviser les lois et les mesures administratives entravant en pratique la tolérance religieuse. Nicolas II prend même la peine d'expliciter personnellement ce nouveau cours de la politique religieuse dans une lettre à Pie X du 8/21 janvier 1905 : «La tolérance religieuse est un principe consacré en Russie par les traditions politiques et les mœurs nationales, et Nous avons jugé utile de confirmer récemment ce principe ${ }^{9}$.

Dès mars 1905, une circulaire organise l'application de l'oukase de décembre $1904^{10}$ en autorisant la fondation de confraternités, les processions extérieures, normalisant les déplacements des ecclésiastiques, réorganisant les relations entre pouvoir épiscopal et pouvoir séculier quant aux nominations et transferts $\mathrm{du}$ clergé : autant de changements de taille pour le catholicisme qui sont très bien

4. Cette question fait l'objet de plusieurs mémorandums du Saint-Siège: 10.02.1903, 19.11.1903, 24.03.1904 (ASV, AES III Polonia, fasc. 1, pos. 2, 3, 4).

5. Lettre de Pie X à Nicolas II, 16.12.1904 (ASV, AES III Russia, fasc. 289, pos. 893, f. 56-59, original français).

6. Alexis Strycek, «La révolution de 1905 et les libertés religieuses », in François-Xavier Coquin, Céline Gervais-Francelle, éds., 1905 : La première révolution russe, Actes du colloque du 2-6 juin 1981, P. : Publications de la Sorbonne-Institut d'études slaves, 1986, p. 49. Oukase du 26 février 1903 in Polnoe sobranie zakonov', n $^{\circ} 22.581$.

7. Lettre de Pie X à Nicolas II, 16.12.1904 (document cité note 5). Un cadeau pour la naissance d'Alexis accompagne cette lettre : une image de la Vierge bénie par Pie X.

8. Polnoe sobranie zakonov“" $\mathrm{n}^{\circ} 25.495, \S 6$.

9. Lettre de Nicolas II à Pie X, 08/21.01.1905 (ASV, Segr. Stato, rub. 268, 1905, fasc. unico, f.5rv)

10. Circulaire N. 1891, 24.03/06.04.1905, du ministère de l'Intérieur - Département des affaires ecclésiastiques des confessions étrangères qui détaille, à l'intention des gouverneurs locaux, la suppression des dispositions qui limitaient la liberté religieuse (ASV, AES III Russia, fasc. 292, pos. 906, f. 3-6, trad. italienne). 
accueillis par les catholiques russes et par Rome. Des signes concrets de détente (y compris en Pologne où, au printemps 1905, le nouveau gouverneur général visite une église catholique pendant une messe ${ }^{11}$ ) poussent, par ailleurs, le Saint-Siège à adopter une attitude conciliante et patiente.

\section{Le Dimanche sanglant (9/22 janvier 1905) : la défense de l'autorité}

Cette bienveillance s'exprime nettement à l'occasion du Dimanche sanglant, où la presse vaticane adopte une attitude en décalage par rapport à celle de l'opinion occidentale.

L'Osservatore Romano réduit ces troubles à des «aberrations »12, à une manipulation du peuple par des révolutionnaires professionnels ${ }^{13}$. Toutefois, cette vision romaine du Dimanche sanglant ne fait pas l'unanimité au sein du monde catholique. Ainsi La Croix exprime-t-elle sa sympathie pour le mouvement populaire russe, qu'elle croit sincère : «on a persuadé le tsar qu'ils n'étaient que les délégués de révolutionnaires, quand ils étaient bien les délégués du peuple ${ }^{14}$. Si La Civiltà Cattolica déplore le bilan humain « douloureux au plus haut point » de « ces jours néfastes » qui vont « laisser une longue traînée de haines », elle en profite pour critiquer la presse qui en a fait des « rapports plus ou moins passionnés » qui ne permettent pas «d'éclairer les vraies responsabilités ${ }^{15}$. De même, $L$ 'Osservatore Romano critique les « mille légendes ${ }^{16}$ nées en Europe sur le Dimanche sanglant et fait une chronique amère des «manifestations anti-russes », c'est-à-dire des manifestations de soutien aux révoltés russes en Italie et en France ${ }^{17}$.

Au-delà de la courtoisie de circonstance à l'égard d'un régime avec lequel Rome cherche à améliorer ses relations, il faut voir ici, face à la violence révolutionnaire, l'affirmation d'un réformisme autoritaire qui estime nécessaire la « défense de cette autorité qui n'est pas toujours innocente d'erreurs, mais qui ne peut être impunément détruite sans que naissent centuplés ces dommages que l'on veut éviter ${ }^{18}$.

11. Signe de détente favorablement noté par L'Osservatore Romano, 45 (70), 25.03.1905, p. 1, trad. de l'it : «C'est la première fois, depuis la révolution de 1831, qu'un Gouverneur assiste à l'office catholique ».

12. L'Osservatore Romano, 45 (20), 25.01.1905, p. 1.

13. L'Osservatore Romano [45 (26), 01.02.1905, p. 1, trad. de l'it.] insiste sur les responsabilités des organisateurs « qui rassemblent inconsidérément les masses et (eux restant en sécurité) les entraînent ou à la désillusion ou à l'abattoir ».

14. La Croix, 26 (6689), 25.01.1905, p. 1.

15. La Civiltà Cattolica, 56-1 (1311), p. 361-362, trad. de 1'it.

16. L'Osservatore Romano, 45 (26), 01.02.1905, p. 1, trad. de l'it.

17. L'Osservatore Romano, 45 (25), 31.01.1905, p. 2 et ibid. 45 (26), 01.02 .1905 , p. 1.

18. L'Osservatore Romano, 45 (26), 01.02.1905, p. 1, trad. de l'it. 


\section{L'oukase de tolérance religieuse (17/30 avril 1905) et ses effets : « la joyeuse aurore du catholicisme en Russie ${ }^{19}$ ?}

\section{L'oukase d'avril 1905 : un texte enthousiasmant}

Informé dès le soir du 17/30 avril par un télégramme de l'archevêque de Mohilev²0, le Saint-Siège manifeste au plus haut niveau sa satisfaction. En effet, avant même l'explicitation officielle de l'oukase par voie diplomatique (note du 28 juin 190521), le pape Pie X exprime sa satisfaction, à titre privé, dans une lettre à Nicolas II du 23 mai 1905 : «La joie dont cet acte de Votre Majesté a inondé Notre cœur est d'autant plus grande, qu'il Nous permet d'espérer avec plus de confiance [...] [qu']Elle voudra aussi, pour les catholiques [...] supprimer toute mesure d'exception en leur accordant une entière et sainte liberté $»^{22}$. Après une attente de plusieurs mois ${ }^{23}$, cette satisfaction s'exprime publiquement dans la lettre Poloniae Populum du 3 décembre $1905^{24}$ : «Nous voulons féliciter publiquement votre très puissant empereur [...] d'avoir, par un édit du 30 avril dernier, [...] assuré aux peuples ses sujets la liberté de conscience. Cette concession, qui a réjoui tous les cœurs, a reçu une confirmation et une extension dans un second édit daté du 30 octobre ${ }^{25}$.

Cet enthousiasme se traduit sur le terrain proprement religieux par une mobilisation des milieux unionistes. Ainsi, le père Tondini (barnabite, spécialiste des questions slaves) qui avait déjà adressé le 15 avril 1905 à Pie X une supplique pour diffuser à l'échelle universelle la prière pour l'union de l'Église gréco-russe, déjà en usage dans sa congrégation ${ }^{26}$, n'hésite-t-il pas à réitérer sa demande le 27 mai $1905^{27}$, visiblement autant encouragé par une audience avec le pape que par les événements russes.

Cet enthousiasme romain demeure toutefois ancré dans une diplomatie pragmatique (en juillet 1905, la Secrétairerie d'État cherche à sceller dans des accords bilatéraux l'œuvre accomplie par l'empereur ${ }^{28}$ ) et limité par la prudence des spécialistes catholiques de la Russie. Ainsi, l'un eux, le père Aurelio Palmieri, nuance dès le printemps 1905 l'apport de l'oukase d'avril : «La liberté de conscience a été

19. La Civiltà Cattolica, 56-3 (1324), 19.08.1905, p. 507, trad. de l'it.

20. ASV, AES III Russia, fasc. 292, pos. 906, f. 10.

21. Ibid, f. 20-22.

22. ASV, AES III Russia, fasc. 289, pos. 893, f. 61-62, original français.

23. Cette prise de position publique est à l'étude depuis l'été 1905, poussée notamment par Mgr Casimir Skirmunt (ASV, AES III Russia, fasc. 296, pos. 908, f. 15-17).

24. Nous utilisons l'édition française de 1907 (désormais Poloniae Populum, éd. 1907) in Pie X, Actes de S.S. Pie X, tome II, P. : Questions actuelles, 1907, p. 106-117.

25. Poloniae Populum, éd. 1907, p. 116-117.

26. ASV, AES III Russia, fasc. 290, pos. 898, f. 50-55.

27. Ibid., f. 56.

28. ASV, AES III Russia, fasc. 291, pos. 901, f. 12 : lettre de Merry del Val au Ministre de Russie, 15.07.1905. 
proclamée officiellement en Russie [...] pour qui connaît la bureaucratie russe, même les oukases impériaux, bien que promulgués d'une façon solennelle, ne sont pas toujours appliqués ${ }^{29}$. Selon lui, « le parti réactionnaire » et « les convulsions politiques de la Pologne russe» peuvent enrayer encore longtemps la mise en œuvre de la liberté religieuse ${ }^{30}$.

\section{Les conséquences pratiques de l'oukase d'avril pour l'Église catholique en Russie}

Plusieurs points de l'oukase donnent satisfaction aux catholiques : facilités pour la construction de lieux de culte (point 13) ${ }^{31}$, langue maternelle dans le catéchisme (point 14), mais le point le plus important est la dépénalisation de la sortie de l'orthodoxie (point 1) qui débouche sur des conversions de masse au catholicisme dans les provinces occidentales de l'Empire, dès le printemps 1905. D'après Alexis Strycek, qui se fonde sur des sources officielles, ces sorties de l'orthodoxie vers le catholicisme pour la période 1905-1907 concerneraient 170936 personnes $^{32}$.

L'ampleur de ce mouvement, révélateur d'un potentiel pour le catholicisme, suscite à Rome un intérêt pour la Russie qui demeure mêlé de questionnements car ces conversions posent un véritable problème, à la fois canonique et missionnaire. En effet, parmi les convertis de 1905, beaucoup sont d'anciens « uniates » (ou descendants d'uniates) passés à l'orthodoxie lors des réunions en masse de 1839 et 1875 qui, cette fois, passent au catholicisme, mais de rite latin, ce qui est contraire au droit canonique ${ }^{33}$. Toutefois, le Saint-Siège, par le biais de la Congrégation de la propagation de la foi, agit de façon pragmatique : l'état du clergé russe de rite oriental ne permettant pas d'encadrer cette masse de fidèles, il opte pour le passage des convertis au rite latin, en simplifiant considérablement la procédure du changement de rite (simple demande écrite à l'évêque sans passer par Rome) ${ }^{34}$.

Ce passage généralisé au rite latin ne satisfait pas tous les catholiques intéressés par la question russe : le métropolite Szepticky (archevêque de Lemberg de rite oriental) présente à Rome dès juin 1905 un projet de renaissance de l'Église orientale en Russie ${ }^{35}$, qui séduit profondément les milieux romains (et même un prélat

29. Bessarione, 9 (84), mai-juin 1905, p. 267, trad. de l'it.

30. Ibid., p. 272.

31. Nous utilisons le texte français du Messager officiel (ASV, AES III Russia, fasc. 292, pos. 906, f. 7-8).

32. Strycek, « La révolution de $1905 \ldots$... p. 50.

33. Pour le droit canon catholique, c'est le rite du père qui est déterminant (quelque soit le rite du ministre qui a baptisé ou celui de l'église où a eu lieu le baptême) et le rite demeure valable à vie. Seul le Saint-Siège peut donner l'autorisation de changer de rite (ces principes sont rappelés par Léon XIII dans sa lettre apostolique Orientalium dignitas du 30.11.1894).

34. Lettre de la Congrégation de la Propagation de la foi au secrétaire de la Congrégation des Affaires ecclésiastiques extraordinaires, 11.05.1905 (ASV, AES III Russia, fasc. 290, pos. 897, f. 41).

35. Lettre de Mgr Szepticky, 30.06.1905 (ASV, AES III Russia, fasc. 294, pos. 907, f. 3). 
polonais plutôt hostile au rite oriental comme Mgr Skirmunt ${ }^{36}$ ) par son potentiel missionnaire. Finalement, la renaissance du catholicisme de rite oriental sur les marges occidentales de l'Empire n'aboutit pas dans le contexte de 1905. Par contre, dès 1908, des communautés catholiques de rite oriental se développent à SaintPétersbourg et Moscou, mais à partir de convertis venus de familles orthodoxes et avec l'aide de religieux missionnaires occidentaux ${ }^{37}$.

\section{Rome face à la violence révolutionnaire : l'appel à l'ordre (fin 1905-1907)}

\section{Incertitudes politiques et sociales au lendemain du manifeste d'octobre}

Après le manifeste d'octobre (17/30 octobre 1905), la satisfaction née de ce nouvel acte libéral est assombrie par des inquiétudes sur l'évolution de la situation. Ainsi, La Civiltà Cattolica doute-t-elle de la capacité de la Russie à se démocratiser : « un parlement russe serait une confusion digne de Babel sous l'aspect ethnologique, linguistique, religieux et politique. Les Juifs, les Polonais, les Mahométans, les Arméniens, les Géorgiens vont-ils prendre sérieusement la décision de travailler ensemble avec les Russes pour reconstruire l'organisme endolori et blessé du grand Empire ? Nous en doutons $»^{38}$. De plus, la continuation des violences après le manifeste d'octobre contribue à présenter les Russes comme un peuple immature qui abuse des bontés du tsar : pour L'Osservatore Romano, la terreur répandue dans la population civile par les révolutionnaires à Cronstadt en novembre 1905 «montr[e] au monde entier comment en Russie on sait profiter de la liberté concédée par le tsar ${ }^{39}$. D'une façon plus générale, le désordre nourrit la peur d'une contagion de la révolution et de la violence à l'échelle internationale, de la Russie vers l'Europe occidentale ${ }^{40}$.

36. Mgr Skirmunt, mémoire confidentiel, 12.07.1905 (ASV, AES III Russia, fasc. 294, pos. 907, f. 39-40, original italien).

37. Cf. pour Saint-Pétersbourg : Antoine Wenger, Rome et Moscou, 1900-1950, P.: Desclée de Brouwer, 1987, p. 57-96.

38. La Civiltà Cattolica, 57-1 (1333), 06.01.1906, p. 119-120, trad. de l'it.

39. L'Osservatore Romano, 45 (261), 12.11.1905, p. 1, trad. de l'it.

40. À l'occasion des événements d'Odessa, L'Osservatore Romano critique la presse libérale et les gouvernements occidentaux qui «regardent avec une tranquillité affectée la révolution interne de la Russie, comme une chose qui ne peut pas les atteindre, eux qui croient posséder dans les constitutions modernes un antidote puissant contre toute sorte de soulèvement prolétarien. Tout ceci constitue pour nous une très grave erreur » $(45$ (151), 02.07.1905, p. 1, trad. de l'it). 


\section{Poloniae Populum (3 décembre 1905) : condamnation réactionnaire de la révolte ou refus réformiste de toutes les violences?}

C'est dans ce contexte troublé que la lettre Poloniae Populum est adressée aux évêques de la Pologne russe. D'emblée controversé ${ }^{41}$, ce document pontifical est resté dans les mémoires pour son appel au respect de l'ordre, entrant ainsi dans une « légende noire » des condamnations romaines des révoltes nationales polonaises et, plus généralement, comme un symbole du caractère réactionnaire de la papauté, allié des puissants de la terre. Qu'en est-il vraiment?

Effectivement, l'appel aux catholiques à «se maintenir dans la paix et dans l'ordre ${ }^{42}$, en refusant les partis violents, est bien le point central du document. À la suite de l'archevêque de Varsovie (qui appela en mars ${ }^{43}$ et en décembre $1905^{44}$ à cesser les grèves et à respecter le droit de propriété), Pie X semble bien se poser en fossoyeur du mouvement social en critiquant les grèves « si fréquentes et si préjudiciables au bien public $»^{45}$. Le pape se place ici dans la lignée de la Doctrine sociale de l'Église promue sous Léon XIII refusant les grèves ${ }^{46}$ mais plaidant pour la création d' « associations » afin d'améliorer le sort « des ouvriers et des prolétaires ${ }^{47}$. L'année 1905 marque ainsi le début d'une «action catholique » moderne dans l'Empire, comme à Vilna où, autour de l'évêque, Mgr de Ropp, sont fondés un quotidien, deux hebdomadaires ${ }^{48}$ et un parti politique «baptisant ${ }^{49}$ les mots d'ordre socialistes. Par ailleurs, cette lettre présente une condamnation des partis promoteurs d'un « radicalisme national » qui se livrent à des «crimes horribles dont rougiraient les nations barbares elles-mêmes. Tel [...] le massacre récent des juifs au grand jour, massacre réprouvé et condamné par la loi évangélique, qui nous ordonne d'aimer tout le monde indistinctement $»^{50}$.

41. Cette légende noire, qui cherche à dissocier l'opinion catholique polonaise, voire certains évêques, de la déclaration du pape, se développe en Pologne et en Europe occidentale (cf. le démenti de L'Osservatore Romano 46 (31), 07.02.1906, p. 1).

42. Poloniae populum, éd. 1907, p. 113.

43. L'Osservatore Romano, 45 (56), 09.03.1905, p. 1-2.

44. Lettre pastorale Ad populum polonum, 06.12.1905 (ASV, AES III Russia fasc. 293, pos. 907, f. 80-83).

45. Poloniae populum, éd. 1907, p. 113.

46. Encyclique Rerum Novarum (15.05.1891) : à propos des grèves « À cette maladie si commune et en même temps si dangereuse, il appartient au pouvoir public de porter un remède » (http://www.vatican.va/ holy_father/leo_xiii/encyclicals/documents/hf_l-xiii_enc_15051891_rerum-novarum_fr.html).

47. Poloniae populum, éd. 1907, p. 113. On peut rappeler aussi l'encyclique Il fermo proposito adressée aux évêques italiens le 11.06.1905, consacrée à l'action catholique.

48. Lettre de Mgr de Ropp, 08.01.1906 (ASV, AES III Russia, fasc. 294, pos. 907, f. 57-59).

49. Lettre de Mgr de Ropp, 21.03/03.04.1907, original français (ASV, AES III Russia, fasc. 300, pos. 918, f. 24-32 : «Pour arrêter le mouvement révolutionnaire [...] je promulguais un programme politico-social catholique qui entravât le travail des socialistes »).

50. Poloniae populum, éd. 1907, p. 111. 
Finalement, on ne peut réduire l'appel à l'ordre de Pie X à une simple défense des pouvoirs constitués. L'incitation à l'action sociale et la condamnation sans ambiguïté des pogromes incitent à voir dans Poloniae Populum une expression d'un catholicisme intégral dont le principe est, selon la devise de Pie X («instaurare omnia in Christo»), de christianiser mouvements sociaux et nationaux; le respect de l'ordre étant compris comme une garantie pour les personnes et les biens, un premier pas vers le respect des principes du christianisme.

\section{Les deux premières Doumas (janvier 1906 - été 1907)}

De la fin de l'année 1905 et jusqu'à l'élection de la troisième Douma, la presse vaticane essaie de tenir un juste milieu entre incitation aux réformes ${ }^{51}$ et critique des excès provoqués par le libéralisme en 1905, notamment «la liberté de la presse venue accroître le désordre des esprits $\gg^{52}$. Dans cette dynamique modératrice, ce n'est qu'avec le résultat des élections de la troisième Douma que le quotidien officieux du Saint-Siège s'estime rassuré : « les électeurs russes ont trouvé en euxmêmes la force de se détacher des partis extrêmes $»^{53}$.

Cette modération est de mise au moment où les réformes concernant le catholicisme entrent en application, comme le règlement en 1906 de la question des séminaires de Pologne sur la base de l'accord russo-vatican de $1897^{54}$. D'autre part, les incertitudes de la période incitent à l'attentisme. Face au mouvement de réforme dans l'Église russe, le Saint-Siège reste impassible, simple observateur lointain ${ }^{55}$. La question du rétablissement du patriarcat ${ }^{56}$ est ainsi particulièrement ambivalente pour les catholiques : un patriarcat russe sera-t-il l'occasion de renouer des liens sur une base canonique traditionnelle (et non plus sur le modèle hérité de Pierre le Grand soumettant l'Église aux laïcs) ou bien sera-t-il un concurrent, un «pape russe $»^{57}$ ?

51. « Il serait également impossible, à un gouvernement civilisé de refuser aux peuples certains droits [...] conséquences nécessaires de la vie civile moderne. Tout cela Nicolas II l'a finalement compris en entreprenant des réformes, ou plutôt en les "reprenant" parce que déjà les fondateurs de sa dynastie les avaient commencées » (L'Osservatore Romano, 46 (114), 17.05.1906, p. 2, trad. de l'it.)

52. La Civiltà Cattolica, 57-1 (1333), 06.01.1906, p. 120, trad. de l'it.

53. L'Osservatore Romano, 47 (259), 06.11.1907, p. 1, trad. de l'it.

54. Cf. Mémorandum de la Légation russe du 7/20.04.1906 sur les changements de la situation religieuse (ASV, AES III Russia, fasc. 292, pos. 906, f. 33-36).

55. On trouve sur ce thème de nombreuses études de qualité dans les revues d'érudition unioniste réalisées par des clercs russisants (A. Palmieri dans Bessarione, Gustave Morel dans la Revue catholique des Églises).

56. Revue catholique des Églises, 2 (4), avril 1905, p. 245-248; Bessarione 10 (88), janv.-fév. 1906, p. 42-47.

57. La Civiltà Cattolica, 57-1 (1333), 06.01.1906, p. 123, trad. de l'it. 


\section{Vers une valorisation de la révolution de 1905 (à partir du second semestre 1907)}

L'échec patent des réformes religieuses et les problèmes des catholiques de l'Empire russe (notamment en Pologne, comme en 1909-1912 sur la question de Chelm) constituent progressivement « 1905 » et son édit de tolérance en référence idéale pour la diplomatie du Saint-Siège à l'égard de la Russie.

\section{L'étiolement des acquis de 1905}

Dès l'été 1905, un règlement pour l'application de l'oukase d'avril en restreint le point $1^{58}$. En effet, pour valider une sortie de l'orthodoxie, il faut la signaler, par une procédure relativement complexe, aux autorités civiles et religieuses orthodoxes. Ce règlement, muni d'un effet rétroactif, concerne les dizaines de milliers de personnes converties depuis avril. Appliqué avec fermeté à la fin des années 1900, il débouche sur de nombreux procès, avec de lourdes conséquences locales. Ainsi en 1910, dans le diocèse de Vilna, 27 prêtres catholiques ont été destitués par le gouvernement pour ne pas avoir appliqué le règlement d'août $1905^{59}$. La question des conversions de 1905 demeure jusqu'en 1917 un sujet de conflit permanent entre l'État russe et les catholiques. C'est ainsi que la revue Correspondance de Rome peut écrire en 1911 : «nous n'avons pas dit que - malgré l'oukase - il y a la persécution : nous avons dit que l'oukase lui-même est devenu un instrument de persécution, aux mains savantes de la bureaucratie russe ${ }^{60}$.

Par ailleurs, le droit de conversion est limité en septembre 1907 par la circulaire du Saint-Synode empêchant l'abjuration de l'orthodoxie des militaires actifs ${ }^{61}$. De même, en 1911, plusieurs circulaires ministérielles limitent le point 13 sur la construction de lieux de cultes ${ }^{62}$.

58. Règlement du ministère de l'Intérieur du 18/31.08.1905 (n. 4628) in ASV, AES III Russia, fasc. 311, pos. 933, f. 19-21 (russe).

59. Mgr Michalkiewicz, «Memoriale in causa convertendorum Schismaticorum in dioecesi Vilnensi », 1910 (ASV, AES Rapporti delle sessioni, N 1164a, 1912. Ponenza: Sommario Num I, p. 36-37).

60. «La persécution contre les catholiques dans l'Empire russe » in Correspondance de Rome, 18.11.1911.

61. Rapport «Russie. Limitations illégales très récentes du fameux "Édit de tolérance religieuse” de 1905 », 04.10.1907, italien (ASV, AES III Russia, fasc. 292, pos. 906, f. 39-40).

62. Les circulaires du 19.03.1911 (n.2416) limitant cette liberté à la seule restauration d'édifices et du 20.09.1911 (n.8430) empêchant la récolte de fonds pour les constructions sont traduites et présentées par Mgr Skirmunt dans un rapport du 06.12.1912 (ASV, Seg. Stato, rub. 284 , 1911, fasc. 2 , f. 124 sq.). 


\section{De la critique de la réaction à la mobilisation des catholiques occidentaux}

Si la violence politique individuelle (attentats) fait l'objet d'une dénonciation régulière ${ }^{63}$, la presse vaticane condamne également la violence de l'État (répression policière et judiciaire). La Civiltà Cattolica déplore le bilan humain de la révolution en 1906 (sentences capitales pour des prisonniers politiques «probablement innocent $[\mathrm{s}]$ » $^{64}$ ) et en montre l'absurdité : « à moins de décapiter les trois quarts des sujets russes, il est impossible que la monstrueuse bureaucratie continue à tenir en esclavage des millions d'hommes qui voudraient simplement jouir de quelques libertés ${ }^{65}$. À l'occasion de l'attentat manqué contre Witte début 1907 , la presse vaticane souligne l'hypocrisie de l'État, qui laisse s'exprimer, dans des « articles incendiaires » la violence réactionnaire et antisémite d'extrême-droite ${ }^{66}$.

De cette critique de la réaction, les organes catholiques n'hésitent pas à passer à des campagnes de presse en Occident pour combattre la «persécution » de leurs coreligionnaires russes. Ces campagnes se déploient par vagues en $1908^{67}$, mais surtout en 1911-191268, en $1914^{69}$ et mobilisent revues scientifiques et quotidiens d'opinions. En 1912, par exemple, cette campagne obtient un certain retentissement en Europe occidentale ${ }^{70}$ ainsi qu'en Russie ${ }^{71}$.

La militance obstinée du Saint-Siège pour la liberté religieuse en Russie suscite alors l'ironie de quelques contemporains ${ }^{72}$. Pour comprendre ce paradoxe d'un

63. Après l'attentat manqué contre Stolypin, La Civiltà Cattolica évoque le « règne féroce de la terreur » [57-3 (1350), 15.09.1906, p. 750-751].

64. La Civiltà Cattolica, 58-2 (1364), 20.04.1907, p. 247, trad. de l'it.

65. Ibid. p. 245.

66. La Civiltà Cattolica, 58-2 (1364), 20.04.1907, p. 247, trad. de l'it.

67. A. Pamieri, Bessarione, 13 (103-105), juillet-décembre 1908, p. 151-175.

68. La Civiltà Cattolica, 63-1 (1477), 06.01.1912, p. 49-61 (sur le cathéchisme) ; Ibid. 63-2 (1485), 04.05.1912, p. 346-353 (Lituanie) ; Ibid. 63-2 (1488), 15.06.1912, p. 656-683 (langue russe à l'église).

69. Par exemple, la campagne menée par La Croix en février-mars 1914 : presque quotidiennement, les lecteurs trouvent en première page, sous le titre « La Russie contre le catholicisme » de courts articles donnant des exemples de persécution, extraits de quotidiens polonais.

70. Ainsi un article du journal L'Italie (L'Italie, 02.08.1912, n.215, p. 1 : « La persécution des catholiques en Russie est-elle vraiment ce que dit la "Civiltà Cattolica" ? ») se montre-t-il particulièrement critique et finit-il par rejeter dans le domaine des « exagérations panpolonisantes » les faits avancés par les journaux catholiques.

71. «Le catholicisme en Russie est-il persécuté ? Réponse à la "Revue contemporaine" de Pétersbourg » [La Civiltà Cattolica, 63-3 (1490), 20.07.1912, p. 129-145] répond à l'article de N. Svetovidoff - du bureau des cultes étrangers - paru dans la Revue contemporaine.

72. L'Italie [02.08.1912, n. 215, p. 1, original français, « La persécution des catholiques en Russie est-elle vraiment ce que dit la "Civiltà Cattolica" ? »] fait dire à un « russe catholique » : « En Russie, on applique les lois canoniques sanctionnées par Rome même et la Curie Romaine a bien tort de reprocher aux Russes ce qu'elle a toujours fait avec acharnement [...] Si le Vatican requiert la tolérance pour lui, il devrait aussi l'accorder aux autres, précisément au nom des principes de liberté de conscience qu'il ne cesse d'invoquer ». 
Vatican autoritaire et libéral à la fois, il faut se placer dans une logique où le libéralisme n'est que sélectif, déterminé par les intérêts supérieurs de l'Église ${ }^{73}$.

\section{La mémoire de l'oukase de tolérance : vers une diplomatie nostalgique}

Après 1905, l'oukase de tolérance constitue un argument de négociation paradoxal dans les relations Russie-Saint-Siège. L'oukase est d'abord un argument aux mains de la diplomatie russe pour obtenir des concessions du Saint-Siège. Ainsi, lors de négociations en 1906, l'édit d'avril est rappelé pour obtenir l'abrogation du « décret du Saint-Office en date du 11 juillet 1877 qui interdit l'usage de la langue russe dans le culte supplétoire de l'Église catholique romaine ${ }^{74}$. L'efficacité de ce recours n'est pas négligeable puisque, sans abolir le décret du Saint-Office, l'usage du russe - en tant que langue maternelle - est permis dans le culte supplétoire (partie des offices et sacrements qui peut être dite en langue vulgaire) ${ }^{75}$. Mais, en 1912, on observe un retournement argumentatif de l'oukase d'avril, qui passe au profit du Saint-Siège: un mémorandum du Vatican signale les violations de l'oukase et demande que soit tenue la «promesse solennelle» de $1905^{76}$. La négociation échoue en 1912 devant les dénégations du gouvernement russe ${ }^{77}$, mais l'oukase de 1905 ne reste pas moins un jalon essentiel dans la mémoire diplomatique du Vatican. Ainsi, lorsqu'en juin 1917, le Saint-Siège propose au Gouvernement provisoire un mémorandum pour régler les questions concernant le catholicisme, celui-ci fait amplement référence aux «principes modernes dont s'est inspiré l'Édit impérial de $1905 \gg^{78}$.

Si la promulgation de l'oukase d'avril 1905 comble vraiment le Saint-Siège, à partir de l'automne 1905, les milieux romains laissent transparaître leur inquiétude face à une révolution qui s’installe dans la violence. Le Saint-Siège cherche alors à défendre l'ordre social en s'adressant avec autorité aux Polonais tout en tentant de répondre au défi social par des propositions issues de la récente Doctrine sociale de l'Église. Le rejet de la violence, celle des nationalistes et celle des socialistes, celle

73. Si dans les «pays non-catholiques», l'égalité des cultes est juste, dans les «pays catholiques », la vérité ne doit pas être mise à égalité avec l'erreur ( L'Osservatore Romano, 45 (268), 15.11.1905, p. 1, trad. de l'it.).

74. Mémorandum de la Légation de Russie à la Secrétairerie d'État, 7/20 avril 1906 (ASV, AES III Polonia, fasc. 5, pos. 16, f. 19-20).

75. Décision prise par le pape le 6 juillet 1906 à la suite d'une session de la Congrégation des Affaires ecclésiastiques extraordinaires (ASV, AES III Rapporti delle sessioni № 1075, 1906, Procès-verbal, p. 7).

76. Mémorandum du Secrétaire d'État au gouvernement russe, 26.02.1912 (ASV, Segr. Stato, rub. 284, 1912, fasc. unico, f. 138-142).

77. Lettre de la Légation de Russie au Secrétaire d’État, n. 235, 25.10.1912 (ASV, Segr. Stato, rub. 284, 1911, fasc. 1, f. 71-79).

78. Lettre de la Secrétairerie d'État à la Légation de Russie, 23.06.1917 (ASV, AES III Russia fasc. 313 , pos. 935 , f. $78-81)$. 
des révolutionnaires et celle de l'État, semble apparaître alors comme un véritable critère de discernement pour les milieux romains. C'est finalement l'étiolement des réformes et l'exaspération des tensions après 1907 entre le Saint-Siège et la Russie qui conduisent la papauté à développer une mémoire très positive des événements de 1905 tout en cherchant, par la voie diplomatique et par la mobilisation médiatique des catholiques occidentaux, à protéger tant bien que mal le catholicisme latin et oriental - transformé par la révolution de 1905.

Les événements de 1905, qui ont permis de questionner les stratégies missionnaires pour la Russie, contribuèrent ainsi nettement à développer l'intérêt romain pour le monde russe. Quant aux modalités d'ouverture de la Russie au catholicisme, il est certain qu'après l'expérience de 1905, la voie «politique » semble être le moyen le plus efficace d'obtenir rapidement des améliorations pratiques. Mais l'échec politique relatif de la révolution de 1905 constitue une mise en garde envers une confiance excessive accordée à cette voie. Par ailleurs, la voie « religieuse » demeure entrouverte, même si la réforme de l'Église orthodoxe russe s'effectue lentement et ne semble pas opérer de rapprochement significatif avec Rome.

C'est donc dans une triple dynamique d'enthousiasme missionnaire, de réflexion rénovée sur les processus révolutionnaires et enfin d'incertitude sur l'évolution de la Russie que le Saint-Siège aborde les révolutions de 1917.

Laboratoire de recherche historique Rhone-Alpes, Université Lyon 2

Religions, sociétés et acculturations (RESEA)

laura.pettinaroli@laposte.net 